\title{
Los costos de producción agrícola en el marco de la normativa financiera internacional
}

\author{
Grace Tamayo-Galarza'; Carlos Mancheno-Vaca²; Adriana Rodríguez-Herrera³; Marco Posligua-San Martín
}

Fecha de recepción: 23 de Febrero 2018

\section{Resumen}

La constante evolución de la normativa financiera internacional ha tenido un importante impacto en el ámbito de la gestión de costos de producción agrícola, desde su concepción teórica hasta la práctica financiera. En este artículo se pretende reflejar esos cambios mediante el análisis de un caso de aplicación de costos de producción para este sector, emitiendo conclusiones positivas sobre la implementación de la normativa y los avances que se han registrado en las últimas décadas, estableciendo en un resumen de costos de producción, los costos directos, indirectos y generales en la producción para el caso en estudio con datos reales.

Palabras clave: Contabilidad agropecuaria, activos biológicos, inventarios.

\section{Abstract}

The constant evolution of international financial regulations has had an important impact in the field of agricultural production cost management, from its theoretical conception to financial practice. This article intends to reflect these changes through the analysis of a case of application of production costs for this sector, issuing positive conclusions on the implementation of the regulations and the advances that have been registered in the last decades, establishing a summary of production costs, direct, indirect and general costs in production for the case under study with real data.

Keywords: Agricultural accounting, biological assets, inventories.

\section{Introducción}

La agricultura en el Ecuador es una actividad económica importante y socialmente fundamental al usar la mano de obra rural. Los datos agropecuarios que contiene la contabilidad son la base para obtener información acerca del contexto que comprende al proceso de la producción, transformación y gestión estableciendo controles efectivos y reuniendo una adecuada información que sirva a éste para la toma de decisiones. Todo miembro del sector agropecuario debe hacer uso de la contabilidad independientemente de la importancia de su aprovechamiento, para alcanzar un mejor entendimiento de la situación financiera de su actividad económica que le permite tomar decisiones sobre si debe seguir en su actividad presente, diversificarlo, fusionarlo, alquilar o cambiar de operación.

Iniciando el estudio, la NIC 2 INVENTARIOS indica en su objetivo que es prescribir el tratamiento contable de las existencias. Un tema fundamental en la contabilidad de las existencias es la cantidad de costo que debe reconocerse como un activo, y ser diferido hasta que los correspondientes ingresos ordinarios sean reconocidos. Esta Norma suministra una guía práctica para la determinación de ese costo, así como para el posterior reconocimiento como un gasto del ejercicio, incluyendo también cualquier deterioro que disminuya el importe en libros al valor neto realizable. Pero también prescribe dentro de sus excepciones a: (c) los activos biológicos relacionados con la actividad agrícola y productos agrícolas en el punto de cosecha o recolección (véase la NIC 41, Agricultura)

Esta Norma no será de aplicación para la valoración de las existencias mantenidas por: (a) Productores de productos agrícolas y forestales, de productos agrícolas tras la cosecha o recolección, así como de minerales y productos minerales, siempre que sean medidos por su valor neto realizable, de acuerdo con prácticas bien consolidadas en
Fecha de aceptación: 12 de Septiembre 2018 esos sectores. En el caso de que esas existencias se midan al valor neto realizable, los cambios en este valor se reconocerán en el resultado del ejercicio en que se produzcan dichos cambios.

Por tanto, estas existencias a que se ha hecho referencia en el párrafo anterior se valoran por su valor neto realizable en ciertas fases de la producción. Ello ocurre, por ejemplo, cuando se hayan recogido las cosechas agrícolas siempre que su venta esté asegurada por un contrato a plazo sea cual fuere su tipo o garantizada por el gobierno, o bien cuando exista un mercado activo y el riesgo de fracasar en la venta sea mínimo. Esas existencias se excluyen únicamente de los requerimientos de valoración establecidos en esta Norma.

En segundo lugar, el IASB decidió realizar modificaciones a través de las cuales las plantas productoras deben contabilizarse de la misma forma que un activo fijo bajo la NIC 16 PROPIEDADES, PLANTA Y EQUIPO, debido principalmente a lo siguiente: El uso de plantas productoras maduras (disponibles para generar productos agrícolas), es similar al uso de una maquinaria para fabricar bienes: Cuando una planta productora se encuentra madura y está totalmente desarrollada, su transformación biológica ya no es significativa en la generación de beneficios económicos futuros; y debido a que las plantas productoras se utilizan solo para generar productos en varios periodos y pasado ese tiempo son habitualmente erradicadas, los únicos beneficios económicos futuros significativos procedentes de dichas plantas productoras surgen de la venta de los productos agrícolas que éstas generan.

Como punto fundamental del estudio, las normas de contabilidad internacional (NIC) establecieron en la NIC 41 AGRICULTURA que todo tipo de activos biológicos y productos agrícolas son medidos al valor razonable menos los costos de venta. La evaluación de la actividad agrícola en el momento corriente (en el punto de cosecha) también permite que el proceso de transformación sea expresado en una manera inmediata, en orden para proveer la posibilidad de estimar beneficios económicos futuros. Así, el valor razonable es útil aproximación para activos biológicos, con las características de transformación biológica. Pero el valor razonable es fácil de entender cuando los mercados para los activos biológicos se encuentran dinámicos y en acción.

Por otro lado, existen dificultades prácticas con la evaluación cuando no hay mercados activos, como es el caso de Ecuador. Además, hay dificultades en la valoración de activos biológicos separadamente desde otros activos sobre los cuales están localizados, tales como la tierra. Por ejemplo: en el caso de un bosque o un viñedo el valor del mercado es a menudo valorado solamente considerando la tierra. En este caso se permite evaluar el valor presente del efectivo de los activos como un residuo calculando la diferencia de valor presente de la tierra agrícola con cosecha y sin cosecha.

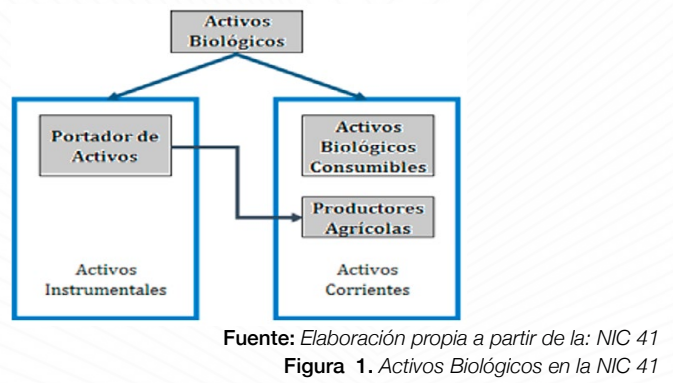

'Candidata a Doctora en Ciencias Administrativas de la UNR, Coordinadora Académica del Programa de Maestría en Administración Tributaria, Profesora del Centro de Economía Pública Instituto de Altos Estudios Nacionales- IAEN. Quito-Ecuador. E-mail: grace.tamayo@iaen.edu.ec

${ }^{2}$ Candidato a Doctor en Ciencias Administrativas de la UNR, Profesor de la Facultad de Ciencias Administrativas, Universidad Tecnológica Equinoccial- UTE. Quito-Ecuador. E-mail: carlos_mancheno79@hotmail.com ${ }^{3}$ Profesora de la Facultad de Ciencias Administrativas, Universidad Tecnológica Equinoccial- UTE. Quito-Ecuador. E-mail: amrodriguez1015@hotmail.com ${ }^{4}$ Profesor del Centro de Economía Pública del Instituto de Altos Estudios Nacionales- IAEN. Quito-Ecuador. E-mail: marco.posligua@iaen.edu.ec 
El proceso de distribución de los costos indirectos fijos a los costos de transformación se basará en la capacidad normal de trabajo de los medios de producción. Capacidad normal es la producción que se espera conseguir en circunstancias normales, considerando el promedio de varios ejercicios o temporadas (Cesaro, Marongiu, Arfini, Donati, Capelli, 2008), dentro de los costos de los productos agrícolas recolectados de activos biológicos de acuerdo con la NIC 41 Agricultura, las existencias que comprenden productos agrícolas, que la entidad haya cosechado o recolectado de sus activos biológicos, se valorarán, para su reconocimiento inicial, por el valor razonable menos los costos estimados en el punto de venta, considerados en el momento de su cosecha o recolección. Este será el costo de las existencias en esa fecha, para la aplicación de la Norma.

Las últimas tres décadas hay un gran incremento en investigaciones sobre el costeo de productos y estimaciones de costos de producción, empezando desde el sector industrial las diferentes metodologías también han sido aplicados en otros sectores, inclusive el sector agropecuario. En la agricultura actual los datos de ingresos y sus resultados son más complejos que en el pasado, la teoría económica se ha vuelto más sofisticada y precisa. ¿Por qué es importante tener información sobre los costos de producción a nivel de hacienda, granja o similares?

Primero, porque las estimaciones de costo del producto son útiles en el proceso de toma de decisiones al nivel analizado: conociendo la utilidad de los productos individuales puede ayudar en la planificación de la producción futura. El costo de los productos puede ser usado para justificación de inversiones, búsqueda de materiales y servicios, introducción de nuevos productos, estrategia de marketing, cambios en procesos de ingeniería y cambios en ingeniería de productos.

Los costos totales y costos variables son también utilizados para evaluar la utilidad de un producto, determinar el proceso de producción óptimo, tomar decisiones sobre la fijación de precios. Comparaciones de la estructura de costos de productos entre unidades productivas (en la misma región o en diferentes regiones) podría también guiar a una mayor eficiencia en el proceso productivo de las unidades productivas individuales. El proceso de benchmarking podría también ser usado para diferentes periodos de tiempo. Segundo, existen diferentes costos para diferentes propósitos, la importancia de usar estimación y cálculo de costos para propósito de formulación de la política está incrementando.

A lo largo del tiempo, los reguladores han usado el costo de producción como base para la formulación de políticas directas o indirectas, especialmente para la toma de decisiones acerca del nivel de apoyo en los precios. A pesar de la importancia de la recolección de información sobre el costo de producción, los métodos contables para las actividades de agricultura han recibido muy poca atención desde los reguladores y contadores en muchos países. En su lugar, algunos países han desarrollado herramientas sofisticadas para la contabilidad en el sector agropecuario (Cesaro et al. 2008)

En febrero de 2001 la NIC 41 AGRICULTURA fue emitida por el International Accounting Standards Board IASB, específicamente para el sector agrícola, la cual prescribe los métodos contables, presentación de estados financieros y la revelación relacionada a la actividad agrícola. Como una consecuencia, muchos estudios y análisis han sido hechos sobre la adopción de las normas emitidas en Estados Unidos y Europa.

La determinación del costo de producción a nivel de unidad productiva, de hecho, requiere una especificación precisa de todos los tipos de datos de la actividad. Algunos costos son específicos, atribuibles directamente a los emprendimientos individuales, mientras otros tienen costos comunes que requieren un procedimiento de asignación. Existen también costos implícitos que deben ser calculados a su valor de oportunidad (mano de obra, capital y tierra). Consecuentemente hay diferentes metodologías para calcular los costos de producción a nivel de unidad productiva, dependiendo de los costos, tipo, enfoque conta- ble, etc. Cada metodología sigue un marco teórico específico y tiene una justificación entre un contexto específico de modelo. La forma en la cual los costos son analizados depende del objetivo final y del uso del análisis.

El 30 de junio de 2015, el Consejo de Normas Internacionales de Información Financiera, emitió varias modificaciones a la NIC 41, que entraron en vigencia el 1 de enero de 2016; sin embargo, son pocas las empresas en el Ecuador que ya la han implementado o están en proceso de hacerlo, teniendo en cuenta que los estados financieros anuales al 31 de diciembre de 2016 debieron presentarse de acuerdo con dicha NIC 41 modificada.

Al respecto, la anterior NIC 41 requería que todos los activos biológicos (incluyendo las plantas productoras), relacionados con la actividad agrícola se midieran al valor razonable menos los costos de venta, basados en el principio de que su transformación biológica (procesos de crecimiento, degradación, producción y procreación), se refleja mejor mediante dicho valor; sin embargo, el IASB observó que esta forma de medición de las plantas productoras no necesariamente iba de la mano con las expectativas de los usuarios de los estados financieros.

Las modificaciones en la NIC 41 tienen por objeto responder a las necesidades y preocupaciones de los usuarios de los estados financieros y, como hemos visto anteriormente, su impacto será sumamente relevante en un sector que es uno de los motores de la economía ecuatoriana y que influye notablemente en todas sus variables micro y macroeconómicas; por lo tanto, las empresas que se dediquen o estén relacionadas al sector agricultura, deberán tomar todas las medidas necesarias para implementar estas modificaciones en la NIC 41 sino lo han realizado ya, al 31 de diciembre de 2016 y de cara a los próximos años, ya que dicho impacto no sólo es contable, sino que también afecta a los principales procesos de negocio de las compañías, control interno, sistemas informáticos y de reporte gerencial, planificación tributaria, entre otros (Mere, 2017).

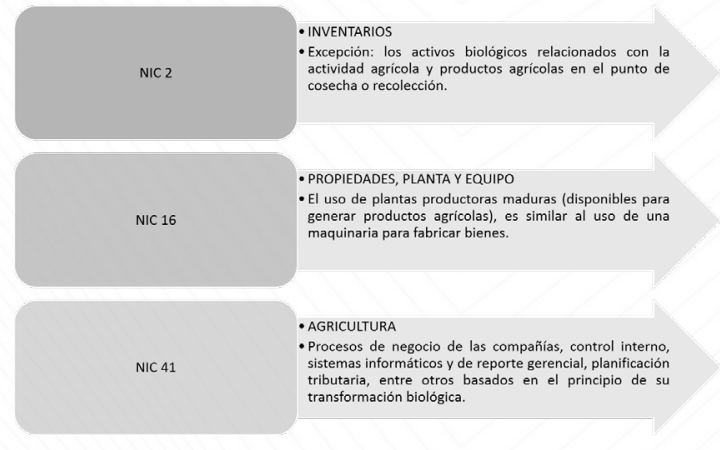

Fuente: Elaboración propia a partir de la NIC 2, 16 y 41 Figura 2. Resumen NIC aplicadas

\section{Metodología}

Partiendo de una realidad que en el Ecuador una gran parte de los actores económicos dedicados al agro no aplican la normativa internacional citada, lo que dificulta la evolución y registro de las actividades inherentes del costeo, hace que el objeto de este estudio sea una respuesta precisa y oportuna para el sector, debido a la metodología siguiente.

Iniciando de la premisa de que el objeto de estudio de la Contabilidad siempre será un hecho económico, se ejecutó un análisis metodológico que parte de una investigación contable estructural, es decir de un profundo análisis de la parte normativa que involucra los procesos de determinación de los costos de producción, en este caso las NIC 2 y 41 , que rige la búsqueda metódica, racional y objetiva de conocimiento que permitió la descripción, explicación y control de la sistemática financiera aplicada.

Luego se procedió a la valoración contable de este hecho económico, aplicando la teoría contable para su abstracción. 
Posteriormente se experimenta con datos reales correspondientes a una granja de producción de cerdos la información financiera determinada en la normativa descrita que comprueba la validez y confiabilidad de la teoría contable, así como el comportamiento de los fenómenos que generan estos datos, mismos que mediante un análisis pertinente al caso bajo estudio permiten finiquitar con reflexiones válidas para casos similares de producción agrícola, en este tercer acápite se verifica el tratamiento que se plantea y fundamenta para el tratamiento del hecho económico abordado.

\section{Resultados}

Los datos obtenidos para el detalle de producción reflejan el estado actual del objeto de estudio.

Tabla 1. Detalle de producción

\begin{tabular}{|lc|}
\hline \multicolumn{1}{c}{ Detalle de producción } & Datos cuantitativos \\
\hline Número de lechones destetados por ciclo & 540 \\
\hline Número de cerdos al finalizar el ciclo engorde & 526 \\
\hline Peso de los cerdos que van al mercado & $100 \mathrm{~kg}$ o 220 libras \\
\hline Total de kg producidos al finalizar & 52.600 \\
\hline La etapa de preparación y manejo & 154 días \\
\hline
\end{tabular}

Fuente: Elaboración propia a partir de estudio

Tabla 2. Detalle de mortalidad por etapa

\begin{tabular}{lcc|}
\hline Tipo de alimento & Edad & Muertos \\
\hline Iniciador & 1 a $2 \mathrm{~m}$. & 3 \\
\hline Crecimiento & 2 a $3 \mathrm{~m}$. & 4 \\
\hline Crecimiento & 3 a $4 \mathrm{~m}$. & 4 \\
\hline Finalización & 4 a $5 \mathrm{~m}$. & 2 \\
\hline Finalización & 5 a $6 \mathrm{~m}$. & 1 \\
\hline Total & & 14
\end{tabular}

Fuente: Elaboración propia a partir de estudio

Tabla 3. Descripción de los costos

\begin{tabular}{lccccccc}
\hline $\begin{array}{c}\text { Tipo de } \\
\text { alimento }\end{array}$ & Edad & $\begin{array}{c}\text { Consumo } \\
\text { por dia }\end{array}$ & $\begin{array}{c}\text { Consumo } \\
\text { por mes }\end{array}$ & $\begin{array}{c}\text { Precio } \\
\text { Unitario } \\
\text { USD }\end{array}$ & $\begin{array}{c}\text { Precio } \\
\text { unitario por } \\
\text { cerdo (USD) }\end{array}$ & $\begin{array}{c}\text { Animales } \\
\text { totales }\end{array}$ & $\begin{array}{c}\text { Resultado } \\
\text { USD }\end{array}$ \\
\hline Iniciador & 1 a $2 \mathrm{~m}$. & $0,3 \mathrm{~kg}$ & 9,00 & 0,28 & 2,52 & 537 & $1.353,24$ \\
\hline Crecimiento & $2 \mathrm{a} 3 \mathrm{~m}$. & $1,6 \mathrm{~kg}$ & 48,00 & 0,26 & 12,48 & 533 & $6.651,84$ \\
\hline Crecimiento & $3 \mathrm{a} 4 \mathrm{~m}$. & $2,3 \mathrm{~kg}$ & 69,00 & 0,23 & 15,87 & 529 & $8.395,23$ \\
\hline Finalización & $4 \mathrm{a} 5 \mathrm{~m}$. & $2,8 \mathrm{~kg}$ & 84,00 & 0,21 & 17,64 & 527 & $9.296,28$ \\
\hline Finalización & $5 \mathrm{a} 6 \mathrm{~m}$. & $3,2 \mathrm{~kg}$ & 96,00 & 0,22 & 21,12 & 526 & $11.109,12$ \\
\hline Total & & & & & & & USD 36.805,71 \\
\hline
\end{tabular}

Fuente: Elaboración propia a partir de estudio

El costo de alimento consumido durante el ciclo productivo es de USD $36.805,71$. Para el costo de producción por $\mathrm{kg}$ de carne de cerdo de esta piara, por concepto de alimento el valor total se divide para los $\mathrm{kg}$ producidos, obteniendo los siguientes resultados:

Ca: USD $36.805,71 / 52.600 \mathrm{~kg}$.

USD $=0,699728327$

Posteriormente se analiza los componentes mano de obra, locales, equipos y maquinarias, determinados en las siguientes tablas:

Tabla 4. Costos de mano de obra

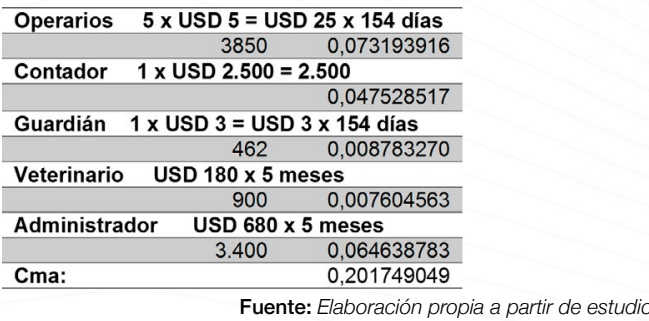

Tabla 5. Locales

\begin{tabular}{l}
$2 \mathrm{~m}^{2} \times 540$ animales $=1.080 \mathrm{~m}^{2}$ \\
$1.080 \mathrm{~m}^{2} \times$ USD $280=$ USD 302.400 \\
$\mathrm{Da}=\underline{\text { USD } 302.400}=$ USD 20.160 \\
\hline 15 años \\
$\mathrm{Dd}=\underline{\text { USD } 20.160}=$ USD $56 \times 154$ días = USD 8.624 \\
\hline 360 días \\
0,163954373
\end{tabular}

Fuente: Elaboración propia a partir de estudio

El costo de producción por kg de cerdo de esta piara, por concepto de locales es de USD 0,163954373

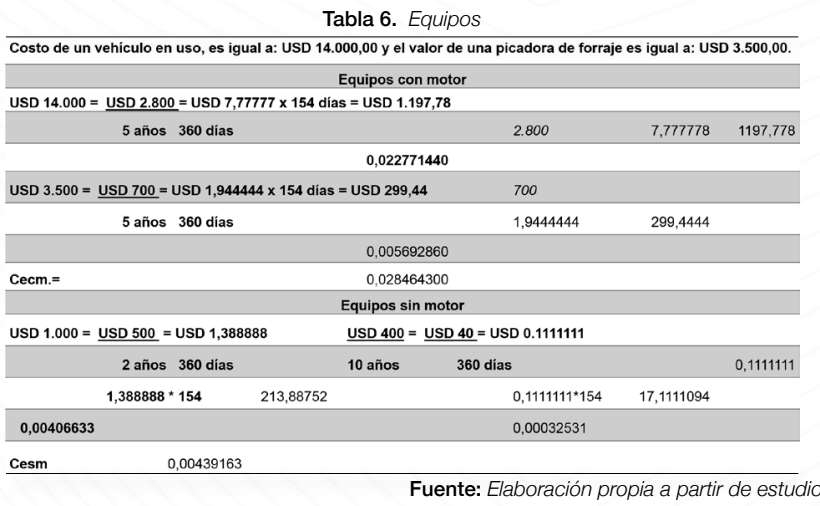

El costo de producción por kg de cerdo de esta piara, por concepto de equipo con motor es igual a USD 0,028464300, mientras que el costo de producción por $\mathrm{kg}$ de cerdo de esta piara, por concepto de equipo sin motor es igual a USD 0,00439163.

Tabla 7. Interés sobre el capital invertido

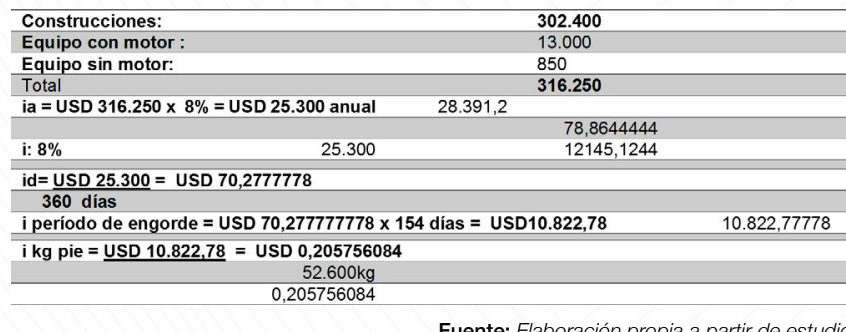

El costo de producción por kg de cerdo de esta piara, por concepto de interés de capital invertido es igual a USD 0,205756084.

Tabla 8. Animales

\begin{tabular}{lr} 
Precio de lechón destetado: USD $30 \times 540=$ USD 16.200 & $\mathbf{1 6 . 2 0 0}$ \\
\hline Can.= USD $16200 / 52600 \mathrm{Kg}=0.30798479$ & 0.307575758 \\
USD 0,30798479 &
\end{tabular}
USD 0,30798479

El costo de producción por $\mathrm{Kg}$ de cerdo de esta piara, por concepto del animal es igual a USD 0,30798479

Fuente: Elaboración propia a partir de estudio

El costo de producción por kg de cerdo de esta piara, por concepto del animal es igual a USD 0,30798479.

Tabla 9. Renta de terrenos

Crt. = USD 150 en el período
USD 0,002525253
$\begin{aligned} & 52.600 \\ & =0,002851711\end{aligned}$

Fuente: Elaboración propia a partir de estudio

El costo de producción por kg de cerdo de esta piara, por concepto de renta del terreno es igual a USD 0,002851711. 


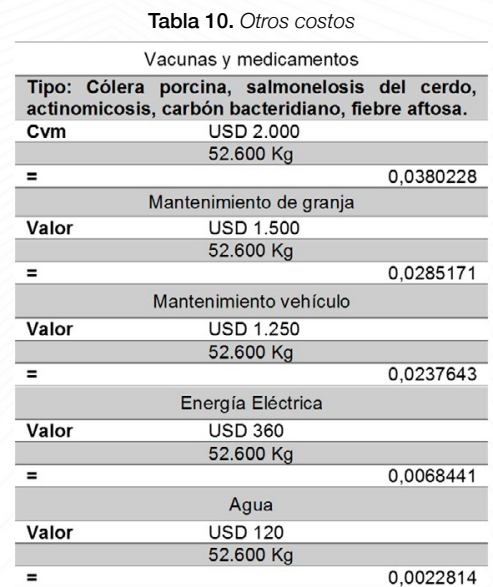

Fuente: Elaboración propia a partir de estudio

Tabla 11. Resumen de costos de producción de un kg de carne de cerdo

\begin{tabular}{|c|c|c|}
\hline \multicolumn{2}{|l|}{ COSTOS DIRECTOS } & \\
\hline \multicolumn{2}{|l|}{ Costo de produccion por cerdo por alimento } & 0,699728327 \\
\hline \multicolumn{2}{|l|}{ Costo de producción por cerdo en Mano de Obra } & 0,081977186 \\
\hline \multicolumn{2}{|l|}{ Costo de producción por cerdo en Animales } & 0,307575758 \\
\hline \multicolumn{2}{|l|}{ Costo de producción por cerdo en vacu. y med. } & 0,038022814 \\
\hline \multicolumn{2}{|l|}{ Costo de producción por cerdo en Mant. Granja } & 0,025252525 \\
\hline \multicolumn{2}{|l|}{ Costo de producción por cerdo en Mant. Vehí. } & 0,023764259 \\
\hline \multicolumn{2}{|l|}{ Costo de producción por cerdo en Luz } & 0,006060606 \\
\hline \multicolumn{2}{|l|}{ Costo de producción por cerdo en Agua } & 0,002281369 \\
\hline \multicolumn{2}{|l|}{ SUBTOTAL } & 1,184662844 \\
\hline \multicolumn{2}{|l|}{ COSTOS INDIRECTOS } & \\
\hline \multicolumn{2}{|l|}{ Costo de produccion por cerdo en Locales } & 0,163954373 \\
\hline \multicolumn{2}{|l|}{ Costo de producción por cerdo en Eq. con motor } & 0.028464301 \\
\hline \multicolumn{2}{|l|}{ Costo de producción por cerdo en Eq. sin motor } & 0,004391635 \\
\hline \multicolumn{2}{|l|}{ Costo de producción por cerdo en Renta de terreno } & 0,002851711 \\
\hline \multicolumn{2}{|l|}{ SUBTOTAL } & 0,199662020 \\
\hline \multicolumn{2}{|c|}{ GASTOS GENERALES DE PRODUCCIÓN } & \\
\hline \multicolumn{2}{|l|}{ Costo de producción por cerdo en Int. De Capital } & 0,205756084 \\
\hline \multirow{2}{*}{\multicolumn{2}{|c|}{$\begin{array}{l}\text { Costo de producción por cerdo en Mano de Obra } \\
\text { (Cont. Vet. Adm.) }\end{array}$}} & 0,119771900 \\
\hline & & (Cont. Vet. Adm.) \\
\hline \multicolumn{2}{|l|}{ SUBTOTAL } & 0,325527984 \\
\hline \multicolumn{2}{|l|}{ TOTAL COSTO DE PRODUCCION $(\mathrm{A}+\mathrm{B}+\mathrm{C})$} & 1,709852848 \\
\hline \multicolumn{2}{|l|}{ INGRESOS: $1 \mathrm{~kg}$ de carne de cerdo } & USD 5,800000000 \\
\hline \multicolumn{2}{|l|}{ UTILIDAD: } & USD 4,090147152 \\
\hline
\end{tabular}

Fuente: Elaboración propia a partir de estudio

\section{Conclusiones}

Al poder analizar los elementos de la presente investigación se puede denotar los siguientes puntos:

La normativa internacional lleva la pauta sobre la determinación de los costos de los activos biológicos, viéndose disminuida la participación de los departamentos contables a nivel latinoamericano.

La valoración de los activos biológicos en muchos de los casos no corresponde a una realidad palpable en nuestro medio, disminuyendo su importancia e impacto sobre las finanzas del sector agropecuario y, por ende, del país.

Es de conocimiento general que valorar los activos biológicos no es tarea fácil. La transformación biológica produce una variedad de cambios de tipo físico - crecimiento, degradación, producción y procreación (NIC $41)$, las cuales no definen un estado concreto del bien en cuestión.

Se conoce que todo bien tiene una vida útil dentro de un período de tiempo determinado. Los activos fijos así llamados son en concreto un ejemplo de bienes que sufren un deterioro a través del tiempo al cual le denominamos depreciación. A pesar de estar sujeto a ello, deterioro a través del tiempo, puede ser remodelado o mejorado según las condiciones del mismo. No sucede lo mismo con los activos biológicos cuyo deterioro, por así decirlo, no se pueden realizar mejoras y por ende está determinado por un tiempo de vida específico.
En vista de las NIC 41, referente al tema agrícola, sustenta el mecanismo de registro y valoración contable del hecho económico objeto del presente estudio, en cuyos resultados sustenta la confiabilidad de la teoría aplicada.

Al realizar los cálculos referentes a la producción de cerdos se pudo observar lo siguiente:

a. Se hizo un análisis de la producción total de kilos llegando a la cantidad de 52.600 dentro de un periodo de 154 días.

b. La mortandad no es significativa dentro de este campo representando cerca del $3 \%$.

c. Se evidencia que al utilizar la teoría contable se puede obtener un costo total de por kilo de 1,709852848 dólares dejando una utilidad por la suma 4.090147152 dólares en la unidad de medida antes especificada.

Cabe mencionar que aplicando la teoría se puede obtener información valiosa que puede ser usada en el ámbito agropecuario en su amplitud. Lo que se esbozó solo corresponde a una pequeña gama, enfocada al sector bovino, la cual puede ser imputado el estudio, lo cual facilitaría al productor el costeo de sus productos, en medidas específicas (tal como en este caso en kilogramos), y con ello fortalecer la toma de decisiones.

Las variables fundamentales para su determinación, sin duda son los costos, pero para la toma de decisiones, en esencia, para producción y utilidad, son sin duda los costos variables en cuyo centro contiene la información práctica que concentra su proyección.

En el Ecuador las prácticas empíricas sin la ayuda de la práctica contable muchas veces hacen que la industria del agro se vea afectado en el mediano y en el largo plazo. Las bases de concentrar el esfuerzo en determinación de costos, en base a unidades que son medibles, proyecta su funcionamiento y ejecución a través del tiempo.

Al realizar el ejercicio en el presente estudio denota un beneficio fundamental para la persona natural o jurídica que direcciona sus esfuerzos al agro, ampliando su concepción, objetivos y estrategias para el manejo de su negocio, facilitando su accionar y en esencia a la toma de decisiones precisas y oportunas en el marco de su desarrollo.

\section{Referencias}

Cesaro, Marongiu, Arfini, Donati, Capelli, 2008, Farm Accountancy Cost Estimation and Policy Analysis of European Agriculture, recuperado de http:// www.s/u.se/facepa

IASB -International Accounting Standards Board, 2001: NIC 41 Agricultura, IASCF cultura, IASCF

IASB -International Accounting Standards Board, 2005: NIC 41 AgriiOS, IASCF

IASB -International Accounting Standards Board, 2005: NIC 2 Inventar-

Mere, 2017, Modificaciones a la norma contable sobre productos biológicos, Perú, https://perspectivasperu.ey.com/2016/07/13/modificaciones-norma-contable-productos-biologicos/ 